\title{
Association of adiponectin, leptin and resistin with inflammatory markers and obesity in dementia
}

\author{
Małgorzata Bednarska-Makaruk • Ałła Graban • Anna Wiśniewska • \\ Wanda Lojkowska • Anna Bochyńska • Magdalena Gugała-Iwaniuk • \\ Ksenia Sławińska • Agnieszka Lugowska • Danuta Ryglewicz • Hanna Wehr
}

Received: 24 November 2016/Accepted: 7 April 2017/Published online: 18 April 2017

(C) The Author(s) 2017. This article is an open access publication

\begin{abstract}
The aim of the study was to determine the role of adiponectin, leptin and resistin in various types of dementia and to investigate their association with inflammatory markers, insulin resistance and abdominal obesity. In 205 patients with dementia [89 with Alzheimer's disease (AD), 47 with vascular dementia (VaD), 69 with mixed dementia (MD)], 113 persons with mild cognitive impairment and in 107 controls serum adiponectin, leptin and resistin levels, proinflammatory [interleukin-6 (IL-6), C-reactive protein (hsCRP) and chitotriosidase] and anti-inflammatory (25-OH vitamin D, HDL-cholesterol and paraoxonase 1) markers, as well as glucose metabolism parameters (glucose, insulin and HOMA-IR) were determined. In all-cause dementia adiponectin and resistin levels were significantly higher as compared to the controls; leptin levels did not show differences. Higher adiponectin levels concerned $\mathrm{AD}$ and $\mathrm{MD}$, whereas higher resistin- $\mathrm{VaD}$ and $\mathrm{MD}$. After stratification by abdominal obesity the differences in adiponectin levels remained significant in subjects without obesity.
\end{abstract}

M. Bednarska-Makaruk ( $($ ) · A. Wiśniewska ·

A. Ługowska · H. Wehr

Department of Genetics, Institute of Psychiatry and Neurology, Sobieskiego 9, 02-957 Warsaw, Poland e-mail: makaruk@ipin.edu.pl

A. Graban - W. Łojkowska · A. Bochyńska ·

M. Gugała-Iwaniuk · K. Sławińska · D. Ryglewicz

First Department of Neurology, Institute of Psychiatry and

Neurology, Sobieskiego 9, 02-957 Warsaw, Poland
In all-cause dementia negative correlation of adiponectin with obesity, glucose metabolism parameters, IL-6 and hsCRP and positive correlation with HDLcholesterol were found. Positive correlation of resistin with age, IL-6, hsCRP and chitotriosidase and negative correlation with HDL-cholesterol and paraoxonase 1 were stated. We conclude that dementia of neurodegenerative origin is characterized by elevated adiponectin levels, whereas dementia with vascular changes by increase of resistin. Association with inflammatory indicators may suggest the pro-inflammatory role of resistin in the development of dementia, especially in dementia of vascular mechanism. Identification of this novel biomarker may be important in preventing dementia.

Keywords Adipokines - Dementia - Inflammatory markers · Abdominal obesity

\section{Introduction}

With aging, an increased prevalence of a clustering of metabolic abnormalities has been observed. These abnormalities including abdominal obesity, dyslipidemia, hypertension, and insulin resistance are collectively known as metabolic syndrome (MetS). MetS is regarded as a low-grade, systemic, inflammatory condition associated with an increased risk of atherosclerosis, cardiovascular disease and diabetes 
(Grundy et al. 2005). An increasing body of epidemiological evidence suggested that MetS and its individual components could be linked to a risk of developing age-related cognitive decline, mild cognitive impairment, vascular dementia, and Alzheimer's disease. Recently the presence of a "metaboliccognitive syndrome", i.e. a MetS plus cognitive impairment of degenerative or vascular origin disease was proposed (Frisardi et al. 2010; Parimisetty et al. 2016). Accumulating evidence suggests that obesity (particularly central or abdominal obesity) contributes to chronic inflammation, leading to the development of insulin resistance and the metabolic syndrome (Wellen and Hotamisligil 2003).

Individuals with obesity exhibit a dysregulation of adipokines, which are highly bioactive substances secreted by adipocytes and immune cells. An increasing number of adipokines is described in the literature (Deng and Scherer 2010). Adipokines exert pleiotropic effects on different tissues and regulate numerous important physiological functions such as appetite, energy expenditure, insulin sensitivity and secretion, fat distribution, lipid and glucose metabolism, endothelial function, blood pressure, hemostasis, neuroendocrine functions and also immunity (Deng and Scherer 2010; Blüher and Mantzoros 2015).

It is acknowledged now that adipokines have more widespread influence and functionality in the brain than previously thought. The association between adipokines and clinical dementia or cognitive impairment is largely unexplored, despite published epidemiological data supporting associations between obesity and various types of dementia and Alzheimer's disease. (Parimisetty et al. 2016). Several casecontrol and large population based prospective studies have shown an association of lower circulating leptin levels with $\mathrm{AD}$ or general cognitive decline (Lieb et al. 2009; Baranowska-Bik et al. 2015), but some clinical studies found no differences in $\mathrm{AD}$ and controls (Theodoropoulou et al. 2012; Warren et al. 2012; Teunissen et al. 2015). In case of adiponectin the epidemiological link with AD risk is less clear. Some case-control and large follow-up studies have shown an association of higher circulating or cerebrospinal fluid level of adiponectin with increased risk of AD (Une et al. 2011; van Himbergen et al. 2012; Khemka et al. 2014), but other authors found no difference in adiponectin level between patients with $\mathrm{AD}, \mathrm{VaD}$, MCI and healthy controls (Warren et al. 2012; Bigalke et al. 2011; Dukic et al. 2016). In contrast, in the study of Teixeira et al. (2013) decreased adiponectin serum concentrations are associated with MCI and AD.

Experimental studies in cell lines and AD transgenic mice indicated that leptin can affect APP-A $\beta$ trafficking, $A \beta$ accumulation and clearance, $\beta$-secretase expression and phosphorylation of tau protein (Chacrabarti et al. 2015). Contrary to leptin, the role of adiponectin in the brain has not yet been well elucidated. Exogenous adiponectin was reported to be protective against $A \beta$-induced neurotoxicity under oxidative stress conditions in a cell model (Chan et al. 2012). Adiponectin could modulate brain metabolism and sensitivity of insulin regulating memory and cognitive dysfunction (Song and Lee 2013).

Resistin, although classified as an adipokine, in humans is expressed in macrophages and plays important roles in inflammation throughout the body. Its serum level is increased in cardiovascular disease (Karbowska et al. 2009; Reilly et al. 2005), other inflammation-related diseases (Pang and Lee 2006) and in diabetes (Rajala et al. 2004), however, studies relating human resistin to insulin resistance, or diabetes have shown conflicting results.

The impact of resistin on the physiology and the pathophysiology of the central nervous system remains poorly defined. The potential protective effects of resistin against $A \beta$ neurotoxicity in mouse cells which overproduced $A \beta$ was stated by Liu et al. (2013). The results of the two clinical studies are inconclusive. A recent study of Mirabell et al. (2013) suggested that resistin was not related to cognitive function performance, whereas according to another study $\mathrm{AD}$ was associated with increased serum resistin levels (Kizilarslanoglu et al. 2015).

Epidemiological and clinical studies have suggested that chronic inflammation may have an important role in dementia (Solfrizzi et al. 2006; Tan et al. 2007). Several pathways through which inflammation can drive AD pathogenesis have been identified (Lee et al. 2009). For example, increased levels of proinflammatory cytokines can stimulate amyloid precursor protein (APP) processing to generate more $\mathrm{A} \beta$, which not only led to neurodegeneration, but also acts on microglia and astrocytes to further increase inflammation (Blasko et al. 2000). Recently vitamin $\mathrm{D}$, which was stated to be a neuro-immunomodulator, was suggested to play a role in the pathogenesis of various brain diseases (Fernandes de Abreu et al. 
2009). Accumulating evidence indicates that antioxidant and anti-inflammatory properties of high density lipoproteins (HDL) may play significant role in neuroprotection and maintaining cognitive function during aging (Hottman et al. 2014). It was shown that paraoxonase 1 (PON1), an enzyme carried by HDL, mainly contribute to antioxidant and anti-inflammatory properties of the particle (Aharoni et al. 2013). The enzyme chitotriosidase, a nonspecific marker of macrophage activation, is recently considered to be a novel marker of inflammation (Kanneganti et al. 2012).

The aim of the present study was to investigate the serum levels of selected adipokines (adiponectin, leptin and resistin) in various types of dementia and mild cognitive impairment in relation to abdominal obesity - the most important feature of metabolic syndrome. We also explored the association of adipokines with some pro- (interleukin-6, C-reactive protein, chitotriosidase) and anti-inflammatory markers (HDL-cholesterol, paraoxonase 1, 25-OH vitamin D) and insulin resistance indices (glucose, insulin, HOMA-IR index).

\section{Materials and methods}

\section{Subjects}

The investigated group consisted of 425 elderly subjects. The whole group with dementia included 205 consecutive patients ( 75 males and 130 females) aged $74.5 \pm 8.23$ years, admitted to the First Department of Neurology, Institute of Psychiatry and Neurology in Warsaw between June 2012 and December 2015. There were 89 patients (mean age $72.8 \pm 8.13$ years) diagnosed as probable Alzheimer's disease, 47 (mean age $74.9 \pm 9.00$ years) as dementia of vascular origin and 69 (mean age $76.5 \pm 7.39$ years) as mixed dementia. 113 persons aged $70.5 \pm 8.89$ years were recognized as having mild cognitive impairment. 107 persons aged $71.3 \pm 7.95$ years, without any cognitive deficits and in good general health, matched to dementia patients for age, constituted the control group. The control subjects were mainly recruited from healthy volunteers, healthy spouses of demented patients as well as from patients from the outpatient clinic, in whom dementia or MCI were excluded basing on neuropsychological test.

Dementia was recognized according to ICD-10 and DSM-IV criteria. Mini Mental State Examination (MMSE) and a Clock Drawing Test were used as screening tests for existing dementia in all participants. All patients underwent a general physical and neurological evaluation, neuroimaging examinations (computer tomography-CT or magnetic resonanceMRI) and comprehensive neuropsychological assessment. The type of dementia was diagnosed based on the NINCDS-ADRDA scale for AD and NINDSAIREN for $\mathrm{VaD}$. MD was defined as coexisting cerebrovascular disease (CVD) in AD patients, defined as significant radiological evidence of CVD in CT or MRI. For MCI diagnosis, the Petersen criteria were used (Petersen and Negash 2008).

All participants were Polish and were white. Most of the subjects were living in urban area $(87.3 \%$ dementia patients, 95.6\% MCI subjects and 95.3\% controls), mainly in Warsaw and the suburbs. Majority of investigated individuals were home-dwelling persons and only four patients with dementia were nursing-home residents. Over $90 \%$ participants were retired and in most of them physical activity is limited to daily activities.

In all study participants demographic and lifestyle data, anthropometric measures and clinical anamnesis were obtained by physicians and nursing staff. Past and current disease history addressed major agerelated disorders i.e. cardiovascular disease (coronary artery disease including myocardial infarction, stroke or TIA, peripheral artery disease), hypertension, diabetes, hyperlipidemia cancer and depression.

In all subjects current use of medications was recorded including: lipid-lowering (statins and/or fibrates), anti-diabetic (sulfonylureas and/or biguanides and/or insulin), anti-hypertensive (ACE-inhibitors/angiotensin II receptor blockers and/or betablockers and/or calcium channel blockers and/or diuretics) drugs and drugs used in dementia treatment (cholinesterase inhibitors or memantine).

Subjects with evidence of malignant disease, myocardial infarction or stroke up to six months before recruitment, hypothyroidism, alcohol abuse, autoimmune disorders, symptoms of acute infections, therapy with steroids, severe depression and other mood disorders were excluded from the study. 
The study was approved by the Ethics Committee of the Institute of Psychiatry and Neurology. Subjects gave their informed consent either directly or received it from their guardians.

\section{Methods}

The blood samples for biochemical and molecular analyses were obtained after an overnight fast. Serum was isolated and stored at $-70^{\circ} \mathrm{C}$ until analysis. Genomic DNA was extracted from peripheral blood leukocytes by phenol/chloroform extraction and ethanol precipitation (Maniatis et al. 1982).

Body weight and height were measured in the morning with the subject wearing light clothing and without shoes. Body mass index (BMI) was calculated as body weight (in $\mathrm{kg}$ ) divided by height (in meters) square. Waist circumference $(\mathrm{cm})$ was measured while the subjects were standing after normal expiration.

Serum adiponectin, leptin and resistin levels were measured by solid-phase sandwich enzyme immunoassay technique (R\&D Systems Inc., USA). Serum IL-6 (R\&D Systems Inc., USA) and CRP (DRG Instruments $\mathrm{GmbH}$, Germany) were measured by high sensitivity solid-phase ELISA assays. Serum insulin was measured by a solid phase enzyme-linked immunosorbent assay (DRG Instruments $\mathrm{GmbH}$, Germany), based on the sandwich principle. Serum 25-OH Vitamin D was measured by a solid phase enzyme-linked immunosorbent assay (DRG Instruments $\mathrm{GmbH}$, Germany), based on the principle of competitive binding. All ELISA kits were commercially available, and assays were carried out according to manufacturer's instructions.

Leptin to adiponectin ratio (L/A) (which was shown to be a better biomarker for MetS diagnosis criteria than leptin and adiponectin separately) was calculated.

HOMA-IR-homeostatic model assessment index (which was shown to correlate well with insulin sensitivity measured by the euglycemic clamp method) was calculated as follows: fasting glucose $(\mathrm{mmol} / \mathrm{L}) \mathrm{x}$ fasting insulin $(\mathrm{mU} / \mathrm{L}) / 22.5$ (Muniyappa et al. 2008).

Glucose was determined using enzymatic method after an overnight fast and after $75 \mathrm{~g}$ glucose load (oral glucose tolerance test-OGTT). OGTT was not performed in subjects with previously diagnosed diabetes. Diabetes mellitus (DM) was defined according to American Diabetes Association guidelines (2010) by a recorded fasting glucose $\geq 7.0 \mathrm{mmol} / \mathrm{L}(126 \mathrm{mg} /$ $\mathrm{dL}$ ) or post-load/casual blood glucose level of at least $200 \mathrm{mg} / \mathrm{dL} \geq 11.1 \mathrm{mmol} / \mathrm{L}(200 \mathrm{mg} / \mathrm{dL})$ or a previous diagnosis of diabetes mellitus or the use of an oral hypoglycemic agent or insulin.

Serum triglycerides and cholesterol were determined in the fresh serum by enzymatic methods. HDL cholesterol was determined after removing apolipoprotein B containing lipoproteins by precipitation.

Metabolic syndrome was defined by IDF (International Diabetes Federation) and AHA/NHLBI (American Heart Association/National Heart, Lung, and Blood Institute) joint scientific statement criteria (Alberti et al. 2009). The following abnormalities were taken into account: (1) abdominal obesity with waist circumference $\geq 102 \mathrm{~cm}$ in men and $\geq 88 \mathrm{~cm}$ in women; (2) elevated triglyceride (TG): $\geq 150 \mathrm{mg} / \mathrm{dL}$ (1.7 $\mathrm{mmol} / \mathrm{L})$ or drug treatment for elevated TG; (3) reduced HDL cholesterol (HDL-C): $<40 \mathrm{mg} / \mathrm{dL}$ $(1.03 \mathrm{mmol} / \mathrm{L})$ in $\mathrm{men},<50 \mathrm{mg} / \mathrm{dL}(1.3 \mathrm{mmol} / \mathrm{L})$ in women or drug treatment for reduced HDL; (4) elevated blood pressure (BP): systolic $\mathrm{BP} \geq 130 \mathrm{~mm}$ $\mathrm{Hg}$ or diastolic $\mathrm{BP} \geq 85 \mathrm{~mm} \mathrm{Hg}$ or drug treatment for hypertension; (5) elevated fasting glucose $\geq 100 \mathrm{mg}$ / $\mathrm{dL}$ or hypoglycemic treatment. The presence of three out of five abnormal findings constituted a diagnosis of MetS.

Paraoxonase 1 activity was determined spectrophotometrically based on the Kitchen et al. method (1973) using phenyloacetate as substrate. One unit of activity was $1 \mu \mathrm{mol}$ of phenol liberated per minute per $1 \mathrm{~mL}$ of serum.

Serum activity of chitotriosidase was measured by a spectrofluorometric method according to Hollak et al. (1994) using the synthetic substrate 4-methylumbelliferylb-N-N'-N" ${ }^{\prime \prime}$-triacetylchitotrioside (Sigma Chemical Co, St. Louis, MO). Fluorometric measurements were made at excitation $\lambda=365 \mathrm{~nm}$ and emission $\lambda=445 \mathrm{~nm}$ (Hitachi, Japan).

Duplication of 24-bp (dup24 bp) in exon 10 of the chitotriosidase gene (CHIT1) (rs3831317) was identified by duplication mutation analysis using PCR according to Boot et al. (1998). DNA fragments amplified from the normal and mutant alleles, were identified after electrophoresis in 3\% agarose gel. The identification of chitotriosidase variant form is indispensable because it is strongly influencing the activity of the enzyme-homozygotes of the mutant form display extremely low activity (Boot et al. 1998). 
APOE $\varepsilon 2 / \varepsilon 3 / \varepsilon 4$ polymorphism (rs7412 and rs429358) was investigated using the PCR-Restriction Fragment Length Polymorphism (PCR-RFLP) method according to Hixon and Vernier (1990), consisting of amplification of the APOE gene fragment, its enzymatic cleavage by Hin6I restriction enzyme (Fermentas, Lithuania) and identification of DNA fragments after electrophoresis on $10 \%$ polyacrylamide gel.

\section{Statistical analysis}

Statistical analysis was performed using Statistica version 12 (StatSoft). Shapiro and Wilk test was carried out to ascertain the normality of the distribution of continuous variables. Normally-distributed variables were presented as mean $\pm \mathrm{SD}$. Not-normally-distributed variables were presented as geometric means with interquartile ranges (IQR) and were logarithmically transformed to approximate a normal distribution before statistical analysis, but the results were expressed as crude data. Student $t$ test or Oneway Analysis of Variance (ANOVA) followed by Dunnett's post hoc test was carried out to assess differences for continuous variables among subjects with various types of dementia, MCI and controls. The mean levels of adipokines and glucose metabolism parameters were also adjusted for age, sex and BMI and the anti-hypertensive, anti-diabetic and lipidlowering treatment using the covariance analysis (ANCOVA) with above mentioned variables as covariates. The mean levels of hsIL-6 and hsCRP were adjusted for age, current smoking status and the anti-hypertensive, anti-diabetic and lipid-lowering treatment and mean level of $25-\mathrm{OH}$ vitamin D was adjusted for age, sex, season and geographical region (ANCOVA). Factorial Two-way ANOVA was used to evaluate effects of two categorical variables (i.e. the presence of abdominal obesity and dementia diagnosis) on one continuous variable (i.e. each adipokine level). Statistical significance of the differences in the frequencies of qualitative variables was evaluated using Pearson's $\chi^{2}$ test. Pearson correlation coefficients were calculated to describe associations of plasma adipokines with metabolic and inflammatory indices as well as with MMSE score among dementia and control subjects (univariate analysis). The associations between various types of dementia and adipokines were identified using univariate and multiple logistic regression analysis with age, sex, level of education and the presence of the APOE \&4 allele and the use of anti-hypertensive, anti-diabetic and lipidlowering therapy as confounding factors and presented as odds ratios (OR) with $95 \%$ confidence intervals (CI). Multivariate forward stepwise regression analysis was performed to assess the independent effects of the different factors on serum adiponectin, leptin and resistin levels in whole investigated group. The following variables (chosen on the basis of previous univariate analyses) were included in the model: for adiponectin analysis BMI, HDL cholesterol, HOMAIR, hsIL-6, for leptin analysis BMI, HDL cholesterol (HDL-C), HOMA-IR, hsCRP and for resistin analysis HDL cholesterol, IL-6, 25(OH) vitamin D, creatinine, serum chitotriosidase, current smoking. Moreover in all analyses age, sex, anti-hypertensive, anti-diabetic and lipid-lowering therapy and the type of dementia were included.

$\mathrm{p}$ values lower than 0.05 were considered as statistically significant.

\section{Results}

Clinical and biochemical characteristic of the study groups

Demographic and clinical characteristic of the study groups are presented in Table 1. Patients with allcause dementia and particularly with MD were older than controls. There were no significant differences in gender distribution among investigated groups. All patients with dementia $(\mathrm{AD}, \mathrm{MD}$ and $\mathrm{VaD}$ ) were less educated, had worse cognitive performance and showed a higher frequency of the APOE $\varepsilon 4$ allele when compared to the control group. Patients with $\mathrm{VaD}$ had significant higher prevalence of hypertension and cardiovascular disease, whereas in AD the prevalence of cardiovascular disease was significantly lower as compared to controls. Moreover the percentage of $\mathrm{VaD}$ subjects on anti-hypertensive therapy was also significantly higher than controls. There were no significant differences in proportion of current smokers as well as in mean body mass index (BMI) among investigated groups. The prevalence of abdominal obesity was borderline higher in MCI $(63.7 \%)$ as compared to controls $(52.3 \%)$. 


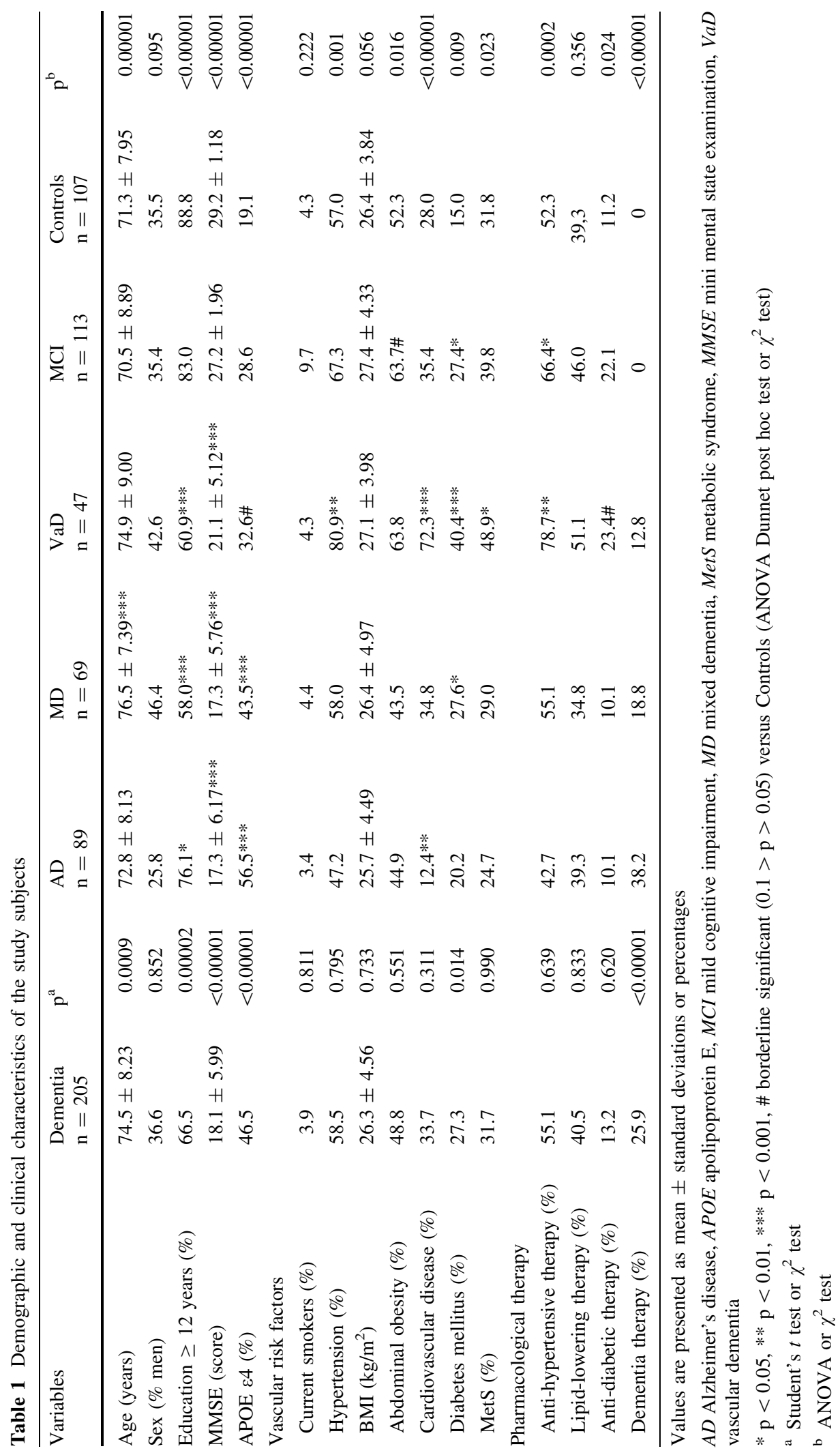


The prevalence of diabetes mellitus was significantly higher in whole group of dementia than in the control group (27.3 vs. $15.0 \%, \mathrm{p}=0.014)$, particularly concerning dementia with vascular changes (VaD and MD) and also MCI. Metabolic syndrome was significantly more frequent only in patients with $\mathrm{VaD}$ (48.9 vs. $31.8 \%$ in controls, $\mathrm{p}=0.023$ ).

Table 2 shows the inflammatory and glucose metabolism markers in various types of dementia, MCI and controls. The whole dementia group in comparison with controls was characterized by statistically significant higher levels of pro-inflammatory IL-6 ( $\mathrm{p}=0.016$ ). Significantly higher levels of IL-6 concerned dementia with vascular component ( $\mathrm{VaD}$ and $\mathrm{MD}$ ). The differences remained significant after adjusting the means for age and smoking using covariance analysis (ANCOVA). There was a tendency towards higher hsCRP levels in mixed dementia group. No significant differences in serum chitotriosidase activity between the investigated groups were found.

There were no significant differences in the levels of anti-inflammatory markers: $25-\mathrm{OH}$ vitamin $\mathrm{D}$, HDL-cholesterol between whole dementia and various types of dementia and the control group. A tendency to higher serum 25-OH vitamin D levels was observed in MCI as compared to controls, the difference reach statistical significance after adjustment the means for age, sex, season, region and BMI. PON1 activity was significantly lower in MD as compared to controls.

Blood glucose both the fasting $(p=0.020)$ and particularly 2-h post load concentrations ( $\mathrm{p}=0.000001)$ were significantly higher in all patients with dementia, but the levels of insulin and insulin resistance index (HOMA-IR) showed no differences as compared to the control group. A significantly higher 2-h post load glucose level was observed in all types of dementia as compared to the control group. There were no statistically significant differences in the levels of fasting glucose, insulin and HOMA-IR between the various types of dementia, MCI and the control group.

Serum adiponectin, leptin and resistin in dementia and healthy controls

The serum levels of investigated adipokines in all studied groups are shown in Table 3 . In the whole dementia group the mean levels of adiponectin $(p=0.002)$ and resistin $(p=0.00009)$ were significantly higher and leptin/adiponectin (L/A) ratio
( $\mathrm{p}=0.015)$ was significantly lower as compared to the control group. In comparison with the controls, significantly higher concentrations of adiponectin were stated in dementia of neurodegenerative origin (AD and MD), and significantly higher levels of resistin were observed in dementia with vascular changes ( $\mathrm{VaD}$ and $\mathrm{MD}$ ). The differences remained significant after adjusting the means age, sex, BMI and anti-hypertensive, anti-diabetic and lipid-lowering therapy using covariance analysis (ANCOVA) with above mentioned variables as covariates. There were no significant differences in leptin levels between whole dementia, different types of dementia and MCI as compared to the control group. The L/A ratio was borderline significantly lower in $\mathrm{AD}$.

To test whether plasma levels of adipokines (leptin, adiponectin and resistin) are independently associated with the presence of various types of dementia, we performed a univariate and multivariate logistic regression analysis including known dementia risk factors such as age, gender, level of education and APOE $\varepsilon 4$ allele as confounding variables (Table 4). All subjects were stratified according to tertiles of adiponectin, leptin, L/A ratio and resistin. Both the crude odds ratios (ORs) and ORs after adjusting for age, sex, years of education and APOE $\varepsilon 4$ allele indicate that higher adiponectin levels were significantly associated with the increased risk of all-cause dementia, AD and MD, but not with VaD and MCI. The all-cause dementia, AD and MD risk were over 3 times greater in third tertile as compared with the first tertile of serum adiponectin levels adjusted. The risk of all-cause dementia and AD decreased progressively with increased L/A ratio from the first to the third tertile (in all-cause dementia adjusted $\mathrm{OR}=0.45$ and in AD OR = 0.35). In the case of resistin crude ORs showed significant association of increased levels of resistin with higher risk of all-cause dementia $(\mathrm{OR}=2.50), \quad \mathrm{MD} \quad(\mathrm{OR}=4.05) \quad$ and $\quad \mathrm{VaD}$ $(\mathrm{OR}=3.02)$. However these associations were no longer statistically significant after controlling for the other risk factors. Logistic regression analysis show no association of leptin levels with risk of dementia.

Influence of abdominal obesity on adipokine levels in dementia and healthy controls

The comparison of investigated adipokines levels between subjects with and without abdominal obesity 


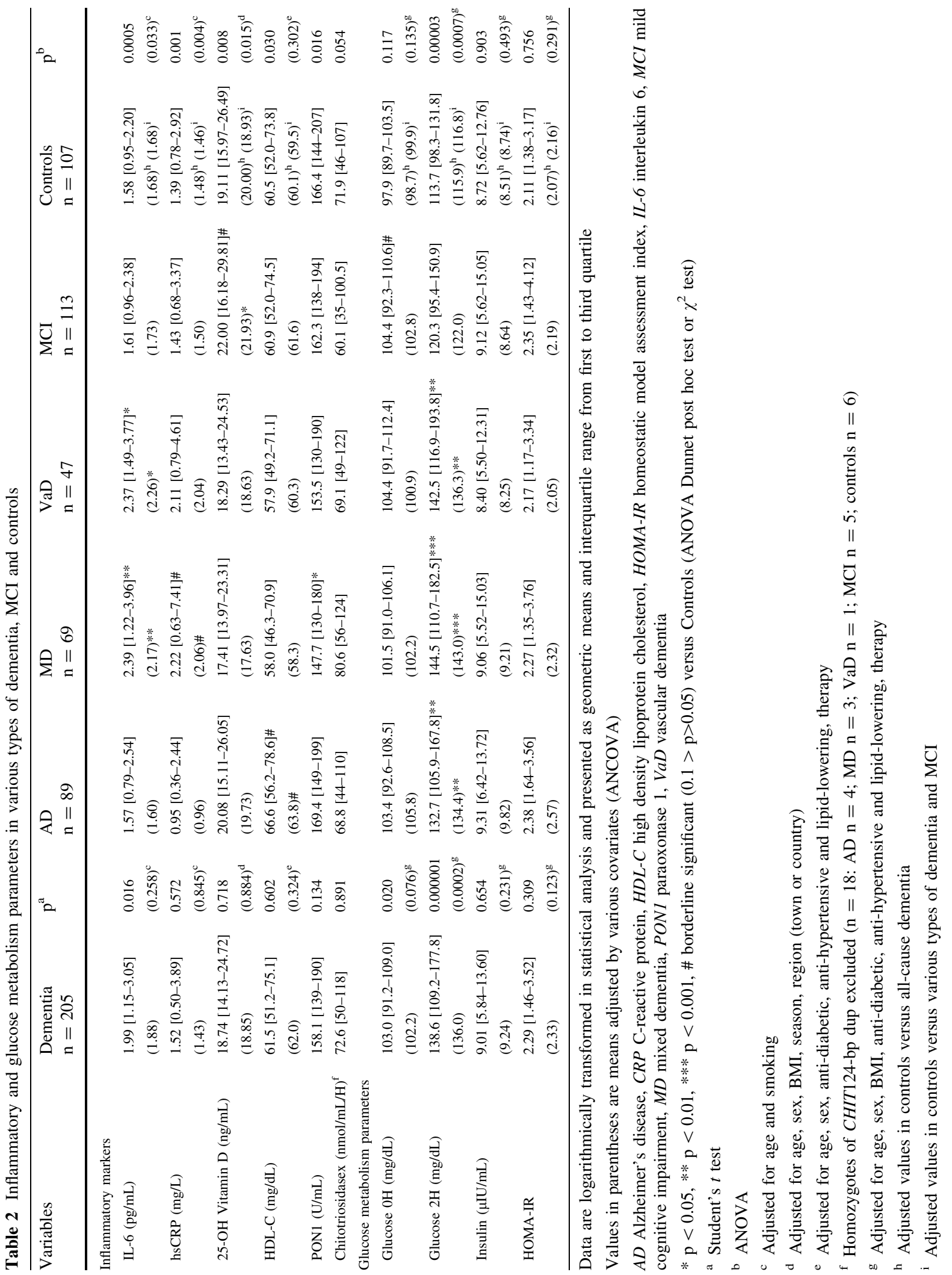




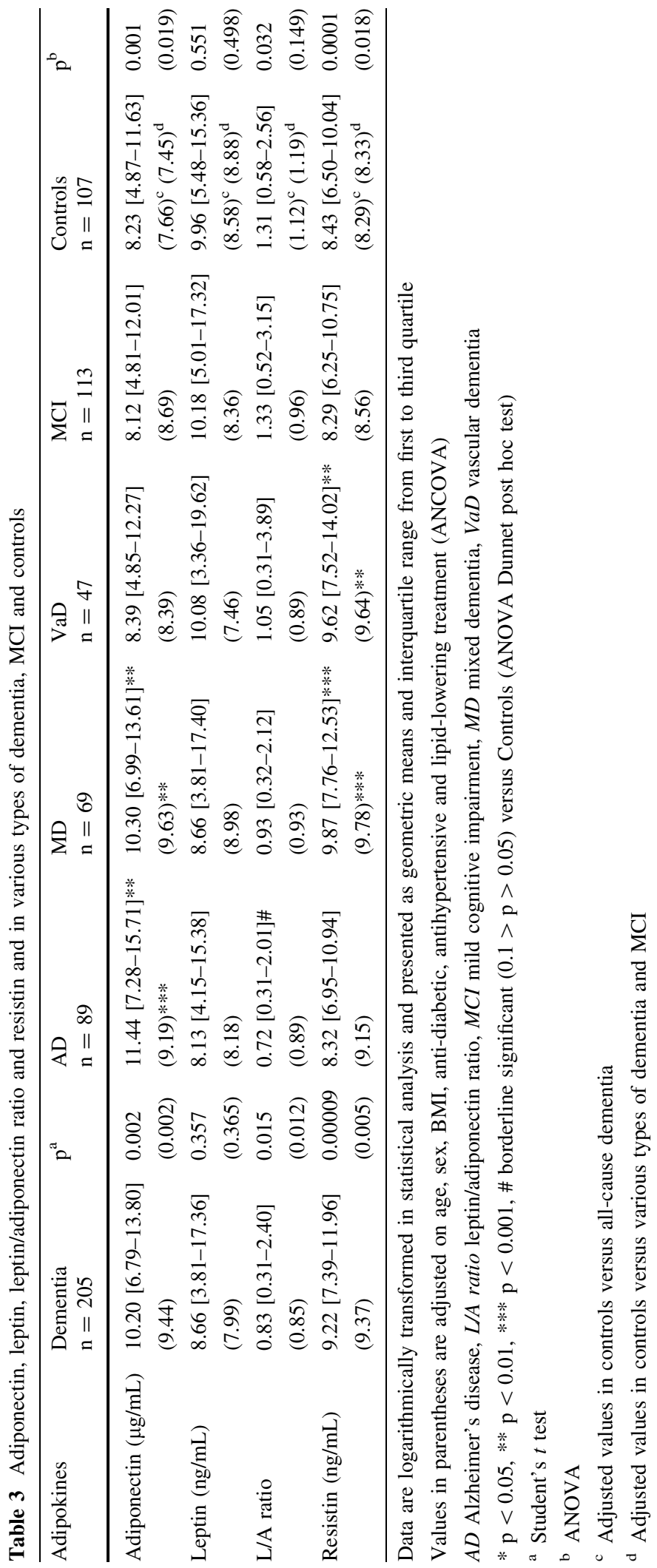




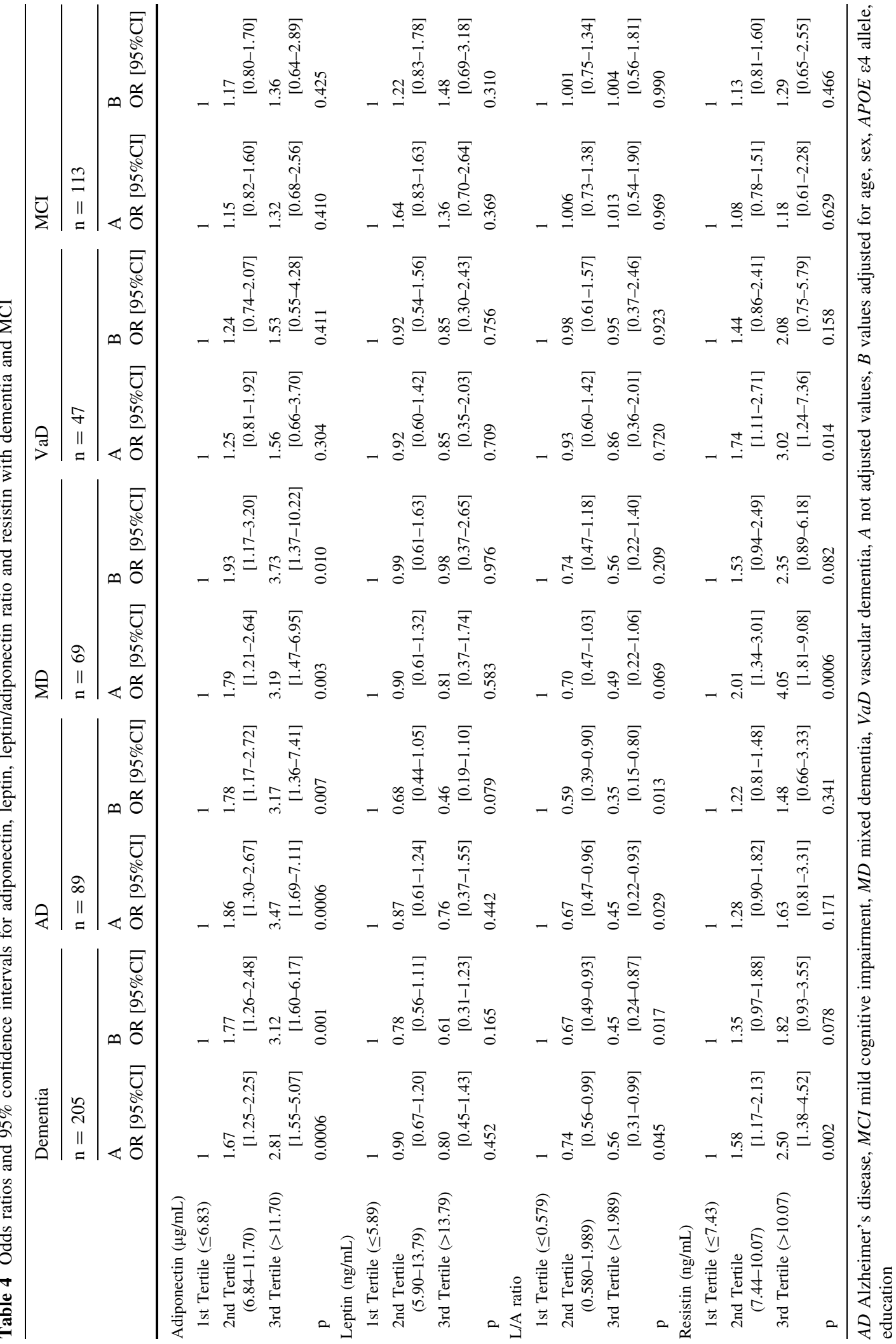



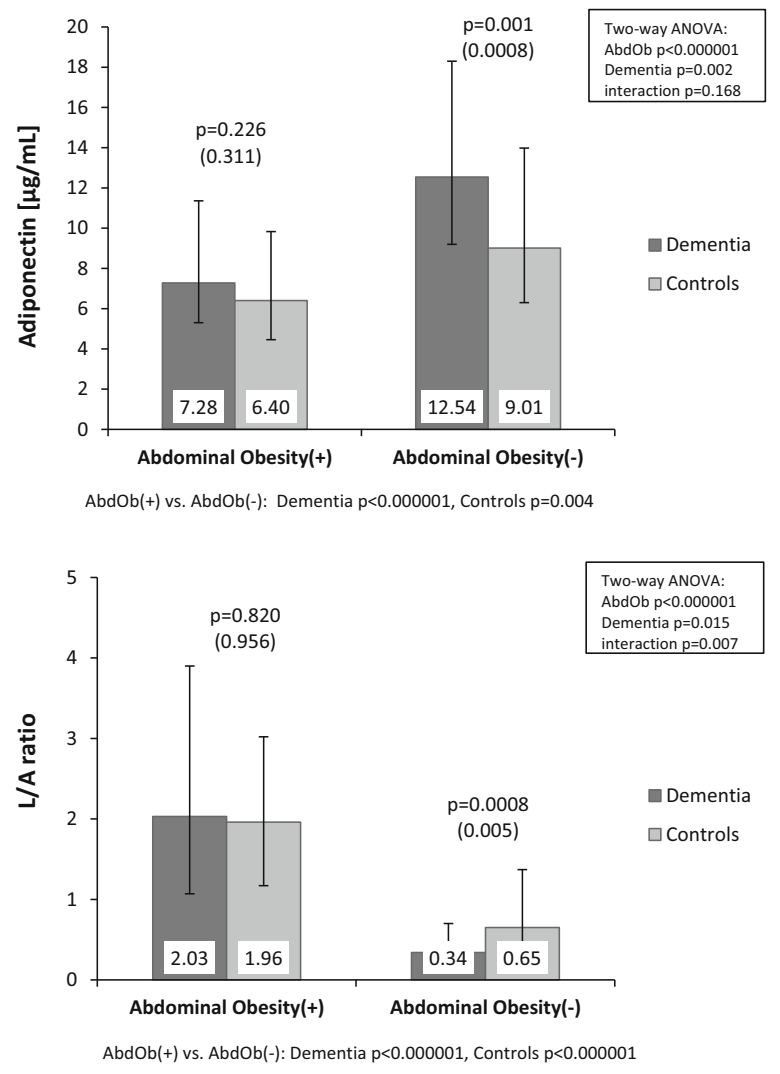

Fig. 1 Serum adiponectin, leptin, resistin levels and leptin/ adiponectin (L/A) ratio according to dementia and abdominal obesity (AbdOb). Data are presented as geometric means with

revealed significant differences concerning adiponectin and leptin but not resistin levels, both in all-cause dementia and controls. Adiponectin levels were significantly lower, while leptin levels were significantly higher in subjects with abdominal obesity (Fig. 1).

Moreover, in all-cause dementia obese patients compared to patients without abdominal obesity significantly higher levels of pro-inflammatory indices (IL-6 and hsCRP) and lower anti-inflammatory parameters (HDL-C) were observed. Higher levels of BMI and parameters associated with glucose metabolism (the post-load glucose, insulin and HOMA-IR) were also found in abdominal obesity. In the control group the relationships were less pronounced (data not shown).

To examine the possible influence of the abdominal obesity on the differences in adipokines levels between all patients with dementia and controls we stratified study subjects into subgroups with and without abdominal obesity (Fig. 1). This allowed to
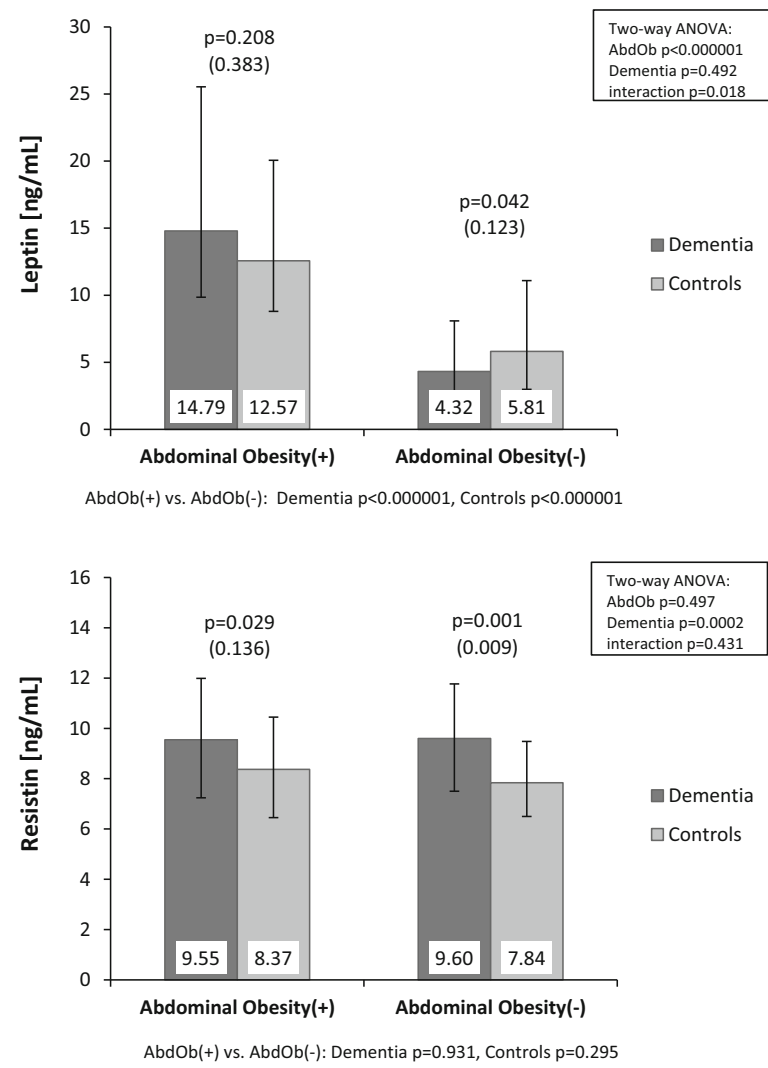

interquartile ranges. Values in parentheses are adjusted on age, sex, anti-diabetic, anti-hypertensive and lipid-lowering treatment (ANCOVA)

demonstrate significant differences in the concentrations of adiponectin between the whole dementia group and controls (i.e. the higher levels of adiponectin in dementia) only in subjects without abdominal obesity. The significantly lower levels of L/A ratio in dementia as compared with controls was observed also only in subjects without abdominal obesity. Interestingly, statistically significant differences in concentrations of leptin between allcause dementia and controls appeared only after the stratification by abdominal obesity. The statistically significant lower level of leptin in all-cause dementia in comparison with controls was found only in those without abdominal obesity $[4.32 \mathrm{ng} / \mathrm{mL}$ (2.33-8.09) vs. $5.81 \mathrm{ng} / \mathrm{mL} \quad(2.98-11.09), \quad \mathrm{p}=$ 0.042]. There was no significant influence of abdominal obesity on resistin levels in the whole dementia group. The higher levels of resistin in dementia were stated both in subjects with and without abdominal obesity. 
The influence of both abdominal obesity and dementia status on serum adipokines levels was confirmed by a Two-way analysis of variance (Fig. 1). The analysis showed significant main effects of both the presence of dementia and the abdominal obesity on adiponectin levels. The level of leptin depended only on the presence of abdominal obesity, but not dementia status. Moreover, in the case of leptin levels a significant interaction between dementia status and abdominal obesity was shown $(\mathrm{p}=0.018)$ indicating the opposite direction in differences of leptin levels in all-cause dementia, dependent on abdominal obesity presence i.e. tendency to higher leptin levels in demented patients with obesity and significantly decreased level in demented patients without abdominal obesity (as compared to appropriate control groups with and without abdominal obesity). Similarly to leptin the L/A ratio depended only on the presence of abdominal obesity, but not dementia status and the significant interaction between dementia status and abdominal obesity was shown $(p=0.007)$. The level of resistin depended only on dementia status, and not on the presence of abdominal obesity.

The similar influence of the presence of MetS on investigated adipokines levels in dementia was observed (data not shown).

Correlations of adipokines with glucose metabolism parameters and inflammatory indices in patients with dementia and controls

The correlation coefficients of adiponectin, leptin and resistin with selected metabolic parameters and inflammatory indices in the entire dementia group and controls are shown in Table 5.

A significant negative correlation of adiponectin levels with BMI and glucose metabolism parameters (fasting glucose, insulin and HOMA-IR index) and with pro-inflammatory indices (IL-6 and hsCRP) and the positive correlation with HDL-cholesterol level (considered as negative indicator of inflammation) was found in the whole dementia group. Borderline negative correlation of resistin with MMSE score (indicating the severity of dementia) was observed. In controls, similar results were obtained, with exception of hsCRP.

In the case of leptin, as compared to adiponectin, the opposite direction of correlations with the same parameters were stated in all-cause dementia i.e. positive correlation with BMI, fasting glucose, insulin, HOMA-IR, IL-6 and hsCRP and positive correlation with HDL-C. In controls similar results were obtained with exception of IL-6 and HDL-C. As expected, the significant negative correlation between leptin and adiponectin was stated in dementia $(\mathrm{R}=-0.327$, $\mathrm{p}=0.000002$; data not shown).

Highly significant positive correlation between the level of resistin and age and indices of inflammation (IL-6 and hsCRP, chitotriosidase activity) and a negative correlation with HDL-cholesterol and PON1 activity was stated in patients with all-cause dementia. Borderline negative correlation was observed with $25-\mathrm{OH}$ vitamin D serum levels. In the control group the significant positive correlation of resistin with age and IL-6 was demonstrated. Moreover, we did not observe any correlation between resistin and adiponectin and leptin, both in whole dementia and control group (data not shown).

Multivariate regression analysis (Table 6) performed in the whole group of investigated individuals demonstrated significant positive associations between adiponectin and HDL-C, age, AD and MD status and negative associations with HOMA-IR, male sex, anti-diabetic therapy and BMI. It was shown that the variables included in the model explained $46.2 \%$ of the variance of serum adiponectin levels. In the case of leptin significant positive associations with BMI, HOMA-IR and hsCRP and negative association with male sex were found. The variables included in the model explained $63.4 \%$ of the variance of serum leptin levels. The analysis showed the significant positive associations between resistin and serum creatinine, age, IL-6 and $\mathrm{VaD}$ and $\mathrm{MD}$ status and negative associations with male sex. It was shown that the variables included in the model explained $21.9 \%$ of the variance of serum resistin levels.

\section{Discussion}

Risk factors for all-cause dementia, AD and dementia with vascular changes in the brain exhibit overlap with those for cardiovascular disease. One of the several important risk factors is metabolic syndrome and abdominal obesity associated with deregulation of adipokines-substances secreted by adipocytes and immune cells (Frisardi et al. 2010; Parimisetty et al. 2016). 
Table 5 Univariate correlation analysis

\begin{tabular}{|c|c|c|c|c|c|c|}
\hline & \multicolumn{3}{|c|}{ Dementia $(n=205)$} & \multicolumn{3}{|c|}{ Controls $(\mathrm{n}=107)$} \\
\hline & Adiponectin & Leptin & Resistin & Adiponectin & Leptin & Resistin \\
\hline \multicolumn{7}{|l|}{ Age } \\
\hline $\mathrm{R}$ & 0.132 & 0.034 & 0.300 & 0.025 & -0.064 & 0.294 \\
\hline $\mathrm{p}$ value & 0.059 & 0.633 & 0.00001 & 0.797 & 0.516 & 0.002 \\
\hline \multicolumn{7}{|l|}{ MMSE } \\
\hline $\mathrm{R}$ & -0.119 & 0.063 & 0.007 & - & - & - \\
\hline $\mathrm{p}$ value & 0.089 & 0.372 & 0.926 & & & \\
\hline \multicolumn{7}{|l|}{ BMI } \\
\hline $\mathrm{R}$ & -0.519 & 0.644 & 0.014 & -0.324 & 0.529 & 0.034 \\
\hline $\mathrm{p}$ value & $<0.000001$ & $<0.0000001$ & 0.837 & 0.0007 & $<0.0000001$ & 0.729 \\
\hline \multicolumn{7}{|c|}{ Glucose $0 \mathrm{~h}$} \\
\hline $\mathrm{R}$ & -0.234 & 0.184 & -0.003 & -0.293 & 0.264 & 0.075 \\
\hline $\mathrm{p}$ value & 0.0007 & 0.008 & 0.971 & 0.002 & 0.006 & 0.442 \\
\hline \multicolumn{7}{|l|}{ Insulin } \\
\hline $\mathrm{R}$ & -0.360 & 0.402 & -0.057 & -0.409 & 0.228 & 0.084 \\
\hline $\mathrm{p}$ value & $<0.000001$ & $<0.0000001$ & 0.415 & 0.00001 & 0.018 & 0.390 \\
\hline \multicolumn{7}{|c|}{ HOMA-IR } \\
\hline $\mathrm{R}$ & -0.402 & 0.415 & -0.046 & -0.457 & 0.278 & 0.099 \\
\hline $\mathrm{p}$ value & $<0.000001$ & $<0.000001$ & 0.514 & 0.000001 & 0.004 & 0.309 \\
\hline \multicolumn{7}{|l|}{ IL-6 } \\
\hline $\mathrm{R}$ & -0.187 & 0.153 & 0.340 & -0.224 & 0.023 & 0.260 \\
\hline $\mathrm{p}$ value & 0.007 & 0.029 & 0.0000001 & 0.020 & 0.812 & 0.007 \\
\hline \multicolumn{7}{|l|}{ hsCRP } \\
\hline $\mathrm{R}$ & -0.170 & 0.314 & 0.234 & -0.096 & 0.205 & 0.048 \\
\hline $\mathrm{p}$ value & 0.015 & 0.00004 & 0.0007 & 0.324 & 0.034 & 0.626 \\
\hline \multicolumn{7}{|c|}{ 25-OH Vitamin D } \\
\hline $\mathrm{R}$ & 0.082 & -0.085 & -0.132 & -0.027 & -0.099 & 0.018 \\
\hline $\mathrm{p}$ value & 0.244 & 0.227 & 0.060 & 0.780 & 0.309 & 0.853 \\
\hline \multicolumn{7}{|l|}{ PON1 } \\
\hline $\mathrm{R}$ & -0.024 & 0.042 & -0.236 & 0.099 & 0.107 & -0.166 \\
\hline $\mathrm{p}$ value & 0.727 & 0.545 & 0.0006 & 0.310 & 0.272 & 0.088 \\
\hline \multicolumn{7}{|l|}{ HDL-C } \\
\hline $\mathrm{R}$ & 0.499 & -0.263 & -0.301 & 0.311 & 0.025 & 0.094 \\
\hline $\mathrm{p}$ value & $<0.0000001$ & 0.0001 & 0.00001 & 0.001 & 0.801 & 0.334 \\
\hline \multicolumn{7}{|c|}{ Chitotriosidase } \\
\hline $\mathrm{R}$ & -0.035 & 0.077 & 0.165 & 0.125 & 0.125 & -0.093 \\
\hline $\mathrm{p}$ value & 0.617 & 0.274 & 0.018 & 0.201 & 0.199 & 0.341 \\
\hline
\end{tabular}

Spearman's correlation coefficients (R) of adiponectin, leptin and resistin level with selected metabolic parameters and inflammation indices in dementia and controls

$B M I$ body mass index, CRP C-reactive protein, $H D L-C$ high density lipoprotein cholesterol, HOMA-IR homeostatic model assessment index, $I L-6$ interleukin 6, MMSE mini mental state examination, PON1 paraoxonase 1 
Table 6 Factors affecting adiponectin, leptin, and resistin levels in whole investigated group $(\mathrm{n}=425)$ based on multivariate stepwise regression analysis

\begin{tabular}{|c|c|c|c|c|c|c|c|c|c|}
\hline \multirow[t]{2}{*}{ Variables } & \multicolumn{3}{|c|}{ Adiponectin $^{\mathrm{a}}$} & \multicolumn{3}{|l|}{ Leptin $^{\mathrm{a}}$} & \multicolumn{3}{|c|}{ Resistin $^{\mathrm{a}}$} \\
\hline & $\beta$ & $\Delta \mathrm{R}^{2}$ & $\mathrm{p}$ & $\beta$ & $\Delta \mathrm{R}^{2}$ & $\mathrm{p}$ & $\beta$ & $\Delta \mathrm{R}^{2}$ & $\mathrm{p}$ \\
\hline HDL-C ${ }^{\mathrm{a}}$ & 0.282 & 0.232 & $<0.000001$ & & & & & & \\
\hline HOMA-IR ${ }^{\mathrm{a}}$ & -0.213 & 0.087 & $<0.000001$ & 0.207 & 0.035 & $<0.000001$ & - & - & - \\
\hline Age & 0.210 & 0.053 & $<0.000001$ & & & & 0.156 & 0.054 & 0.00001 \\
\hline Anti-diabetic therapy & -0.197 & 0.044 & $<0.000001$ & & & & & & \\
\hline Sex & -0.183 & 0.026 & 0.00002 & -0.452 & 0.182 & $<0.00001$ & -0.149 & 0.010 & 0.023 \\
\hline BMI & -0.115 & 0.012 & 0.003 & 0.569 & 0.414 & $<0.00001$ & - & - & - \\
\hline $\mathrm{AD}$ & 0.111 & 0.007 & 0.022 & & & & & & \\
\hline MD & 0.140 & 0.009 & 0.008 & & & & 0.101 & 0.006 & 0.037 \\
\hline $\mathrm{VaD}$ & & & & & & & 0.141 & 0.012 & 0.015 \\
\hline $\mathrm{IL}^{-6^{\mathrm{a}}}$ & & & & - & - & - & 0.123 & 0.022 & 0.001 \\
\hline $\mathrm{hsCRP}^{\mathrm{a}}$ & - & - & - & 0.084 & 0.006 & 0.007 & - & - & - \\
\hline \multirow[t]{2}{*}{ Creatinine } & & & & & & & 0.353 & 0.120 & $<0.00001$ \\
\hline & \multicolumn{3}{|c|}{$\mathrm{R}^{2}$ adj $=0.462, \mathrm{p}<0.00001$} & \multicolumn{3}{|c|}{$\mathrm{R}^{2}$ adj $=0.634, \mathrm{p}<0.00001$} & \multicolumn{3}{|c|}{$\mathrm{R}^{2}$ adj $=0.219 \mathrm{p}<0.00001$} \\
\hline
\end{tabular}

Sex (female $=0 ;$ male $=1)$.

Adiponectin: variables included in the model: age, sex, BMI, HDL cholesterol (HDL-C), HOMA-IR, hsIL-6, cognitive status (AD, $\mathrm{MD}, \mathrm{VaD}, \mathrm{MCI})$ and anti-diabetic, anti-hypertensive and lipid-lowering therapy.

Leptin: variables included in the model: age, sex, BMI, HDL cholesterol (HDL-C), HOMA-IR, hsCRP, cognitive status (AD, MD, $\mathrm{VaD}, \mathrm{MCI})$ and anti-diabetic, anti-hypertensive and lipid-lowering therapy and cognitive status (AD, MD, $\mathrm{VaD}, \mathrm{MCI})$ and antidiabetic, antihypertensive and lipid-lowering therapy.

Resistin: variables included in the model: age, sex, HDL cholesterol (HDL-C), IL-6, 25(OH)vitamin D, creatinine, serum chitotriosidase, current smoking, cognitive status ( $\mathrm{AD}, \mathrm{MD}, \mathrm{VaD}, \mathrm{MCI})$ and anti-diabetic, anti-hypertensive and lipid-lowering therapy

$\beta$ the standard regression coefficient, $R^{2}$ adj the multiple coefficient of determination (adjusted), $A D$ Alzheimer's disease, $B M I$ body mass index, $C R P$ C-reactive protein, $H D L-C$ high density lipoprotein cholesterol, HOMA-IR homeostatic model assessment index, $I L-6$ interleukin 6, MD mixed dementia, MMSE mini mental state examination, $V a D$ vascular dementia

${ }^{a}$ Logarithmically transformed in statistical analysis

The main finding of the present study was to establish the association between dementia and adipokines.

Significantly increased adiponectin levels were observed in all-cause dementia, particularly in dementia of neurodegenerative origin (AD and $\mathrm{MD}$ ) as compared to controls. This observation was confirmed by logistic regression analysis and the multivariate regression analysis. The same results were obtained in previous studies showing an association of higher circulating (or cerebrospinal fluid) adiponectin level with increased risk of AD (Une et al. 2011; van Himbergen et al. 2012; Khemka et al. 2014). On the contrary, other authors Teixeira et al. (2013) observed that decreased adiponectin serum concentrations are associated with MCI and AD, but do not predict cognitive decline in elderly individuals. Recently a study of the high molecular-weight adiponectin
(HMW) level and incident dementia in patients with vascular risk factors showed little association with future dementia (Kitagawa et al. 2016).

Interestingly, the increased adiponectin observed in our study in all-cause dementia was significant only in individuals without abdominal obesity or MetS. In a recently published review, Ishii and Iadecola (2016) speculate that the association of increased plasma adiponectin levels with dementia found in some studies, may be surprising for the first moment, since low adiponectin levels are often associated with obesity-associated disorders and adiponectin is generally considered to have protective properties. However, these studies were conducted predominately in patients with $\mathrm{AD}$, where weight loss is characteristic feature. This condition could be associated with higher circulating adiponectin levels. High circulating adiponectin levels may lead to subsequent resistance to 
adiponectin in a similar fashion to insulin resistance and leptin resistance (Ishii and Iadecola 2016).

In the present study we found only a borderline significant negative correlation between adiponectin and severity of dementia measured with MMSE, which is in agreement with previous studies of Bigalke et al. (2011), who observed an inverse trend to a lower MMSE score with increased plasma levels of adiponectin. In contrast, the Khemka et al. (2014) study demonstrated a positive correlation of adiponectin with MMSE.

In addition, both in dementia and non-demented elderly controls, we observed a high negative correlation between adiponectin and metabolic parameters i.e. BMI, fasting glucose, insulin and HOMA-IR index. Similar observations were previously stated in non-demented subjects (Zoico et al. 2004; Hung et al. 2008). A significantly lower level of adiponectin was associated with the presence of abdominal obesity in all our investigated groups, which was confirmed by a Two-way ANOVA analysis.

We also demonstrated negative correlation of adiponectin with pro-inflammatory indices (IL-6 and hsCRP) and positive correlation with HDL cholesterol, a negative indicator of inflammation. These findings are in agreement with previous observations that adiponectin as an anti-inflammatory factor decreases the expression of pro-inflammatory cytokines and increases the expression of anti-inflammatory molecules (Tilg and Moschen 2006; Hung et al. 2008).

The results of the current study did not show significant differences in the levels of leptin between all-cause dementia, different types of dementia, MCI and the control group of elderly subjects. This observation was confirmed by logistic regression analysis and the multivariate regression analysis. These findings are in agreement with results of some previous studies performed in clinical AD (Theodoropoulou et al. 2012; Warren et al. 2012; Teunissen et al. 2015), but different to results of other authors. The results of two large population based prospective studies provided the evidence for a lower incidence of $\mathrm{AD}$ in elderly individuals associated with higher leptin levels (Holden et al. 2009; Lieb et al. 2009). Several case-control studies showed that $\mathrm{AD}$ patients had significantly decreased plasma levels of leptin compared with healthy controls (Bigalke et al. 2011; Baranowska-Bik et al. 2015).

The interesting observation that higher leptin level was associated with better cognitive function only in subjects without central obesity was stated by
Hazzouri et al. (2013), suggesting that obesity may interfere with the neuroprotective effect of leptin on the brain, possibly by leptin resistance. In another small case-control study Power et al. (2001) showed that below-appropriate-weight patients with dementia, both $\mathrm{AD}$ and $\mathrm{VaD}$, had decreased leptin levels in comparison with appropriate-weight controls. Similarly, in our study we observed significantly lower serum leptin level in whole dementia group in comparison with control subjects only in individuals without abdominal obesity. It was confirmed by high positive correlation of leptin and BMI in all investigated groups.

In our present study we did not reveal any significant correlation between leptin and severity of dementia measured with MMSE, which is in agreement with previous studies of Baranowska-Bik et al. (2015) and Teunissen et al. (2015) performed in patients with $\mathrm{AD}$. In contrast, in other studies the positive (Bigalke et al. 2011) or negative (Khemka et al. 2014) correlation of leptin levels with MMSE was found.

Moreover, both in all dementia patients and nondemented elderly controls, we confirmed a high positive correlation between leptin and selected metabolic parameters i.e. BMI, fasting glucose, insulin and HOMA-IR index previously stated in elderly subjects (Zoico et al. 2004). Significantly higher levels of leptin were associated with the presence of abdominal obesity in all investigated groups, which was confirmed by a Two-way ANOVA analysis.

We also demonstrated a positive correlation of leptin with some pro-inflammatory indices IL-6 and hsCRP and negative correlation with HDL cholesterol considered as a negative indicator of inflammation. These findings are in agreement with previous studies showing leptin to be a pro-inflammatory cytokine (Tilg and Moschen 2006).

Leptin has been demonstrated to have a neuroprotective role against $\mathrm{AD}$ pathology and dementia. One has to mention that leptin was considered to be a potential cognitive enhancer (Harvey et al. 2005). It was shown in experimental studies that leptin reduced both brain $A \beta$ load (Fewlass et al. 2004) and hyperphosphorylated tau in neuronal cells (Greco et al. 2008), thus diminishing two important elements of Alzheimer's disease. Moreover, the results from animal studies demonstrated a role of leptin in 
memory decline and AD pathology, e.g., showing that treatment of transgenic mouse model of Alzheimer's disease with leptin led to a reduction of $A \beta$ levels and memory improvement (Greco et al. 2010). These observations made leptin a potential therapeutic agent (Tezapsidis et al. 2009). It was shown that obese people are likely to develop leptin resistance, the condition which may attenuate the beneficial action of leptin on the brain and may be responsible for the lack of protective effect of leptin on dementia and Alzheimer's disease among obese people. In nonobese people, leptin may exert its neuroprotective effect (Ishii and Iadecola 2016).

The most interesting observation in our study is the presence of elevated levels of resistin in all patients with dementia, particularly in dementia with vascular changes in the brain ( $\mathrm{VaD}$ and $\mathrm{MD}$ ) in comparison with non-demented controls. This observation was confirmed by the multivariate regression analysis. In logistic regression analysis crude ORs showed significant association of increased levels of resistin with higher risk of all-cause dementia, MD and $\mathrm{VaD}$, however these associations were no longer statistically significant after controlling for the other risk factors.

The relation of circulating resistin with dementia was studied by other authors but the results of studies that have since been published are inconclusive. A recent population-based longitudinal study performed in 933 subjects over 50 years with moderate to high vascular risk suggested that resistin was not related to cognitive performance measured in different cognitive domains tests (Mirabell et al. 2013). In another small clinical study performed in $38 \mathrm{AD}$ patients and 32 control subjects increased serum resistin levels were observed to be associated with $\mathrm{AD}$. The authors claimed that resistin is a regulatory marker of inflammation and considered resistin being a predictor of Alzheimer's disease caused by activation of the immune system. Based on high sensitivity, specificity and predictive values the authors tried to apply plasma resistin determination as a diagnostic marker for $\mathrm{AD}$ (Kizilarslanoglu et al. 2015).

In the present study, in opposition to leptin and adiponectin (both in dementia and non-demented elderly controls) we did not observe any correlation between resistin and metabolic parameters i.e. BMI, fasting glucose, insulin and HOMA-IR index. These findings are in agreement with the results of several previous studies performed in various populations
(Lee et al. 2003; Bo et al. 2005; reviewed in Schwartz and Lazar 2011). We also did not demonstrate any association of resistin levels with abdominal obesity, neither in all-cause dementia nor in controls, demonstrated after stratification of the data by abdominal obesity, which was confirmed by a Two-way ANOVA analysis.

In the present study, we did show a positive correlation of resistin with inflammatory indicators (interleukin-6 and CRP) and a negative correlation with anti-inflammatory markers (HDL-C and PON1 activity) in all dementia patients. In non-demented controls, only positive correlation with IL-6 reached statistical significance. These results may suggest that resistin could be a remarkable inflammatory marker in dementia. The exact role of resistin in inflammation was not explained and it is still unclear whether it is a marker of inflammation or a pathogenic factor (Karbowska et al. 2009). Some authors stated that it was secreted as a marker of chronic inflammation (Bo et al. 2005; Olefsky and Glass 2010) and on the other hand it was considered to be a factor causing enhanced production and secretion of pro-inflammatory cytokines (Silswal et al. 2005).

Another interesting finding in our study is the significant positive correlation of resistin level with serum chitotriosidase activity stated in all patients with dementia, particularly in dementia with vascular changes. Chitotriosidase (CHIT1) is an enzyme involved in the degradation of chitin and chitin-like substrates. CHIT1 activity is increased in several lineages of activated macrophages which are involved in both physiological and pathologic processes. CHIT1 expression is altered in a number of noninfectious inflammatory diseases. Being a nonspecific marker of macrophage activation, recently the enzyme is considered as a novel marker of inflammation (Kanneganti et al. 2012). The increased circulating CHIT1 activity is a biomarker of lysosomal storage diseases such as Gaucher's disease and is used to monitor progression or the efficacy of treatment with enzyme replacement therapy (Hollak et al. 1994). Elevated serum CHIT1 activities were also reported in atherosclerosis (both coronary and cerebrovascular), and Alzheimer's disease (Elmonem et al. 2016). The positive correlation between resistin and CHIT1 stated in our study confirm the association of elevated resistin levels with activation of macrophages and inflammation. 
Because a tremendous increase of dementia cases and the lack of its effective treatment there is an urgent need to recognize all the factors accelerating or inhibiting its development. In the present study several important factors present in blood serum were analyzed: the hormones produced by adipose tissue (adiponectin, leptin and resistin), pro-inflammatory and anti-inflammatory markers and glucose metabolism parameters in various types of dementia, mild cognitive impairment and controls to identify a characteristic profile for dementia and its type. The role of adiponectin in dementia with neurodegenerative symptoms was confirmed. A very important observation concerns resistin which was stated to be a significant marker of inflammation in dementia especially in dementia of vascular mechanism. Identification of this novel biomarker may be important in preventing dementia.

Our study has some limitations that are worth noting.

First of all in our sample, the all-cause dementia group patients (particularly the MD) were significantly older than non-demented controls and all groups with dementia were also significantly less educated. As age and education are known to be independent predictive factors for cognitive decline, they may influence some of the other comparisons. For example, our younger non-demented controls might be persons who have not yet developed dementia. Adjustment for age and education level was taken into consideration in ANCOVA and Logistic regressions analyses.

Second, it is known that exercise training could influence the lipid, glucose metabolism and inflammatory markers levels. But the precise information about the physical activity of the participants of our study is lacking and is based only on the neuropsychological anamnesis which showed that over $90 \%$ of participants were retired and in most of them physical activity is limited to daily activities.

Third, the sample size in the case of leptin analyses seems too small to reach statistical significance of the results. The calculated power of the ANOVA test used to compare adiponectin, leptin and resistin levels between the investigated groups was 76.2, $18.5 \%$ and $94.9 \%$, respectively. The calculated power of the test used in analysis for leptin is very low. The reason could be the high within-subject variability of leptin levels observed in all investigated groups. The exceedingly high variability of leptin levels could be the fact that leptin levels may be subject to diurnal variation. However, this does not bias our results since leptin was measured at the same time (fasting in the morning) in all our study participants. Assuming that the power of the test should be at least $80 \%$, calculated size of the groups in the case of leptin ANOVA analysis should exceed 229 individuals in each group. We recommend the design of larger case-control studies to further address this issue.

Fourth, we did not investigate the serum level of specific adiponectin isoforms. These isoforms may have distinct biologic effects, for example, the high molecular weight isoform has higher biological activity than the low molecular weight isoform (Swarbrick and Havel 2008). Therefore, additional studies are necessary to evaluate the role of specific adiponectin isoforms in the development of dementia.

\section{Conclusions}

1. Dementia of neurodegenerative origin (Alzheimer's disease and mixed dementia) is characterized by elevated levels of adiponectin, whereas dementia with vascular changes in the brain (vascular and mixed dementia) is characterized by an increase of resistin.

2. Positive correlation of resistin with inflammation indicators and negative correlation with the compounds with anti-inflammatory properties may suggest the potential pro-inflammatory role of resistin in the development of dementia, especially in dementia of vascular mechanism.

3. The opposite direction of correlations of adiponectin and leptin with inflammatory markers in all-cause dementia confirmed previous findings showing adiponectin to have anti-inflammatory and leptin to have pro-inflammatory properties.

4. Significant associations of abdominal obesity and glucose metabolism parameters with adiponectin and leptin but not resistin levels were stated.

Acknowledgements The study was supported by the Polish National Science Centre Grant No. 2011/03/B/NZ7/04288.

\section{Compliance with ethical standards}

Conflict of interests No conflict of interests exists. 
Research involving human participants The study was approved by the Ethics Committee of the Institute of Psychiatry and Neurology, Warsaw, Poland. Subjects gave their informed consent either directly or received it from their guardians.

Open Access This article is distributed under the terms of the Creative Commons Attribution 4.0 International License (http:// creativecommons.org/licenses/by/4.0/), which permits unrestricted use, distribution, and reproduction in any medium, provided you give appropriate credit to the original author(s) and the source, provide a link to the Creative Commons license, and indicate if changes were made.

\section{References}

Aharoni S, Aviram M, Fuhrman B (2013) Paraoxonase 1 (PON1) reduces macrophage inflammatory responses. Atherosclerosis 228:353-361. doi:10.1016/j.atherosclerosis.2013.03. 005

Alberti KG, Eckel RH, Grundy SM, Zimmet PZ, Cleeman JI, Donato KA, Fruchart JC, James WP, Loria CM, Smith SC Jr (2009) International Diabetes Federation Task Force on Epidemiology and Prevention; National Heart, Lung, and Blood Institute; American Heart Association; World Heart Federation; International Atherosclerosis Society; International Association for the Study of Obesity. Harmonizing the metabolic syndrome: a joint interim statement of the International Diabetes Federation Task Force on Epidemiology and Prevention; National Heart, Lung, and Blood Institute; American Heart Association; World Heart Federation; International Atherosclerosis Society; and International Association for the Study of Obesity. Circulation 120:1640-1645. doi:10.1161/CIRCULATIONAHA.109. 192644

American Diabetes Association (2010) Diagnosis and Classification of Diabetes Mellitus. Diabetes Care 34:S62-S69. doi: $10.2337 / \mathrm{dc} 10-$ S062

Baranowska-Bik A, Bik W, Styczynska M, Chodakowska-Zebrowska M, Barcikowska M, Wolinska-Witort E, Kalisz M, Martynska L, Baranowska B (2015) Plasma leptin levels and free leptin index in women with Alzheimer's disease. Neuropeptides 52:73-78. doi:10.1016/j.npep. 2015.05.006

Bigalke B, Schreitmüller B, Sopova K, Paul A, Stransky E, Gawaz M, Stellos K, Laske C (2011) Adipocytokines and CD34+ progenitor cells in Alzheimer's disease. PLoS ONE 6:e20286. doi:10.1371/journal.pone.0020286

Blasko I, Veerhuis R, Stampfer-Kountchev M, Saurwein-Teiss1 M, Eikelenboom P, Grubeck-Loebenstein B (2000) Costimulatory effects of interferon- $\gamma$ and interleukin- $1 \beta$ or tumor necrosis factor $\alpha$ on the synthesis of A $\beta 1-40$ and A $\beta 1-42$ by human astrocytes. Neurobiol Dis 7:682-689. doi:10.1006/nbdi.2000.0321

Blüher M, Mantzoros CS (2015) From leptin to other adipokines in health and Disease: facts and expectations at the beginning of the 21st century. Metabolism 64:131-145. doi:10.1016/j.metabol.2014.10.016

Bo S, Gambino R, Pagani A, Guidi S, Gentile L, Cassader M, Pagano GF (2005) Relationships between human serum resistin, inflammatory markers and insulin resistance. Int $\mathbf{J}$ Obes (Lond) 29:1315-1320. doi:10.1038/sj.ijo.0803037

Boot RG, Renkema H, Verhoek M, Strijland A, Bliek J, de Meulmeester TMAMO, Mannens MMAM, Aetrs JMFG (1998) The human chitotriosidase gene nature of inherited enzyme deficiency. J Biol Chem 273:25680-25685

Chakrabarti S, Khemka VK, Banerjee A, Chatterjee G, Ganguly A, Biswas A (2015) Metabolic risk factors of sporadic Alzheimer's disease: implications in the pathology, pathogenesis and treatment. Aging Dis 6:282-299. doi:10. 14336/AD.2014.002.eCollection

Chan KH, Lam KS, Cheng OY, Kwan JS, Ho PW, Cheng KK, Chung SK, Ho JW, Guo VY, Xu A (2012) Adiponectin is protective against oxidative stress induced cytotoxicity in amyloid-beta neurotoxicity. PLoS ONE 7:e52354. doi:10. 1371/journal.pone.0052354

Deng Y, Scherer PE (2010) Adipokines as novel biomarkers and regulators of the metabolic syndrome. Ann N Y Acad Sci 1212:E1-E19. doi:10.1111/j.1749-6632.2010.05875.x

Dukic L, Simundic AM, Martinic-Popovic I, Kackov S, Diamandis A, Begcevic I, Diamandis EP (2016) The role of human kallikrein 6 , clusterin and adiponectin as potential blood biomarkers of dementia. Clin Biochem 49:213-218. doi:10.1016/j.clinbiochem.2015.10.014

Elmonem MA, van den Heuvel LP, Levtchenko EN (2016) Immunomodulatory effects of chitotriosidase enzyme. Enzyme Res 2016:2682680. doi:10.1155/2016/2682680

Fernandes de Abreu DA, Eyles D, Féron F (2009) Vitamin D, a neuro-immunomodulator: implications for neurodegenerative and autoimmune diseases. Psychoneuroendocrinology 34(1):S265-S277. doi:10.1016/j.psyneuen.2009.05.023

Fewlass DC, Noboa K, Pi-Sunuyer FX, Johnston JM, Yan SD, Tezapsidis N (2004) Obesity-related leptin regulates Alzheimer's. FASEB J 18:1870-1878. doi:10.1096/fj.04-2572com

Frisardi V, Solfrizzi V, Seripa D, Capurso C, Santamato A, Sancarlo D, Vendemiale G, Pilotto A, Panza F (2010) Metabolic-cognitive syndrome: a cross-talk between metabolic syndrome and Alzheimer's disease. Ageing Res Rev 9:399-417. doi:10.1016/j.arr.2010.04.007

Greco SJ, Sarkar S, Johnston JM, Zhu X, Su B, Smith MA, Tezapsidis N (2008) Leptin reduces Alzheimer's diseaserelated tau phosphorylation in neuronal cells. Biochem Biophys Res Commun 376:536-541. doi:10.1016/j.bbrc. 2008.09.026

Greco SJ, Bryan KJ, Sarkar S, Zhu X, Smith MA, Ashford JW, Johnston JM, Tezapsidis N, Casadesus G (2010) Leptin reduces pathology and improves memory in a transgenic mouse model of Alzheimer's disease. J Alzheimers Dis 19:1155-1167

Grundy SM, Cleeman JI, Daniels SR, Donato KA, Eckel RH, Franklin BA, Gordon DJ, Krauss RM, Savage PJ, Smith SC Jr, Spertus JA, Costa F (2005) Diagnosis and management of the metabolic syndrome: an American Heart Association/National Heart, Lung, and Blood Institute scientific statement. Circulation 112:2735-2752. doi:10.1161/ CIRCULATIONAHA.105.169404

Harvey J, Shanley LJ, O'Mailey D, Irving AJ (2005) Leptin: a potential cognitive enhancer? Biochem Soc Trans 33:1029-1032

Hazzouri AZA, Stone KL, Haan MN, Yaffe K (2013) Leptin, mild cognitive impairment, and dementia among elderly 
women. J Gerontol A Biol Sci Med Sci 68:175-180. doi:10.1093/gerona/gls155

Hixson JE, Vernier DT (1990) Restriction isotyping of human apolipoprotein $\mathrm{E}$ by gene amplification and cleavage with Hha1. J Lipid Res 1:545-548

Holden KF, Lindquist K, Tylavsky FA, Rosano C, Harris TB, Yaffe K (2009) Serum leptin level and cognition in the elderly: findings from the Health ABC Study. Neurobiol Aging 30:1483-1489. doi:10.1016/j.neurobiolaging. 2007. 11.024

Hollak CE, van Weely S, van Oerts MH, Aerts JM (1994) Marked elevated plasma chitotriosidase activity. A novel hallmark of Gaucher disease. J Clin Invest 93:1288-1292

Hottman DA, Chernick D, Cheng S, Wang Z, Li L (2014) HDL and cognition in neurodegenerative disorders. Neurobiol Dis 72:22-36. doi:10.1016/j.nbd.2014.07.015

Hung J, McQuillan BM, Thompson PL, Beilby JP (2008) Circulating adiponectin levels associate with inflammatory markers, insulin resistance and metabolic syndrome independent of obesity. Int J Obes (Lond) 32:772-779. doi:10. 1038/sj.ijo.0803793

Ishii M, Iadecola C (2016) Adipocyte-derived factors in agerelated dementia and their contribution to vascular and Alzheimer pathology. Biochim Biophys Acta 1862:966-974. doi:10.1016/j.bbadis.2015.10.029

Kanneganti M, Kamba A, Mizoguchi E (2012) Role of chitotriosidase (chitinase 1) under normal and disease conditions. J Epithel Biol Pharmacol 5:1-9

Karbowska A, Boratyńska M, Klinger M (2009) Resistin: a pathogenic factor or a biomarker of metabolic disorders and inflammation? Postępy Hig Med Dosw 63:485-491

Khemka VK, Bagchi D, Bandyopadhyay K, Bir A, Chattopadhyay M, Biswas A, Basu D, Chakrabarti S (2014) Altered serum levels of adipokines and insulin in probable Alzheimer's disease. J Alzheimers Dis 41:525-533. doi:10. 3233/JAD-140006

Kitagawa K, Miwa K, Okazaki S, Sakaguchi M, Mochizuki H (2016) Serum high molecular-weight adiponectin level and incident dementia in patients with vascular risk factors. Eur J Neurol 23:641-647. doi:10.1111/ene.12915

Kitchen BJ, Maters CJ, Winzor DJ (1973) Effects of lipid removal on the molecular size and kinetic properties of bovine plasma arylesterase. Biochem J 135:93-99

Kizilarslanoğlu MC, Kara Ö, Yeşil Y, Kuyumcu ME, Öztürk ZA, Cankurtaran M, Rahatli S, Pakaştiçali N, Çinar E, Halil MG, Sener B, Cankurtaran ES, Arioğul S (2015) Alzheimer disease, inflammation, and novel inflammatory marker: resistin. Turk J Med Sci 45:1040-1046. doi:10. 3906/sag-1403-55

Lee JH, Chan JL, Yiannakouris N, Kontogianni M, Estrada E, Seip R, Orlova C, Mantzoros CS (2003) Circulating resistin levels are not associated with obesity or insulin resistance in humans and are not regulated by fasting or leptin administration: cross-sectional and interventional studies in normal, insulin-resistant, and diabetic subjects. J Clin Endocrinol Metab 88:4848-4856. doi:10.1210/jc.2003030519

Lee KS, Chung JH, Choi TK, Suh SY, Oh BH, Hong CH (2009) Peripheral cytokines and chemokines in Alzheimer's disease. Dement Geriatr Cogn Disord 28:281-287. doi:10. $1159 / 000245156$
Lieb W, Beiser AS, Vasan RS, Tan ZS, Au R, Harris TB, Roubenhoff R, Auerbach S, DeCarli C, Wolf PA, Seshadri S (2009) Association of plasma leptin levels with incident Alzheimer disease and MRI measures of brain aging. JAMA 302:2565-2572

Liu J, Chi N, Chen H, Zhang J, Bian Y, Cui G, Xiu C (2013) Resistin protection against endogenous $\mathrm{A} \beta$ neuronal cytotoxicity from mitochondrial pathway. Brain Res 1523:77-84. doi:10.1016/j.brainres.2013.05.041

Maniatis T, Fritsch EF, Sambrook J (1982) Molecular cloning: a laboratory manual. Cold Spring Harbor Laboratory, Cold Spring Harbor, Harbor

Miralbell J, López-Cancio E, López-Oloriz J, Arenillas JF, Barrios M, Soriano-Raya JJ, Galán A, Cáceres C, Alzamora M, Pera G, Toran P, Dávalos A, Mataró M (2013) Cognitive patterns in relation to biomarkers of cerebrovascular disease and vascular risk factors. Cerebrovasc Dis 36:98-105. doi:10.1159/000352059

Muniyappa R, Lee S, Chen H, Qoun MJ (2008) Current approaches for assessing insulin sensitivity and resistance in vivo: advantages, limitations and appropriate usage. Am J Physiol Metab 294:E15-E26. doi:10.1152/ajpendo.00645.2007

Olefsky JM, Glass CK (2010) Macrophages, inflammation, and insulin resistance. Ann Rev Physiol 72:219-246. doi:10. 1146/annurev-physiol-021909-135846

Pang S, Le Y (2006) Role of resistin in inflammation and inflammation-related diseases. Cell Mol Immunol 3:29-34

Parimisetty A, Dorsemans AC, Awada R, Ravanan P, Diotel N, d'Hellencourt CL (2016) Secret talk between adipose tissue and central nervous system via secreted factors-an emerging frontier in the neurodegenerative research. J Neuroinflammation 13:67. doi:10.1186/s12974-0160530-X

Petersen RC, Negash S (2008) Mild cognitive impairment: an overview. CNS Spectr 13:45-53

Power DA, Noel J, Collins R, O’Neill D (2001) Circulating leptin levels and weight loss in Alzheimer's disease patients. Dement Geriatr Cogn Disord 12:167-170

Rajala MW, Qi Y, Takahashi N, Banerjee R, Pajvani UB, Sinha MK, Gingerich RL, Scherer PE, Ahima RS (2004) Regulation of resistin expression and circulating levels in obesity. Diabetes 53:1671-1679. doi:10.2337/diabetes.53.7. 1671

Reilly MP, Lehrke M, Wolfe ML, Rohatgi A, Lazar MA, Rader DJ (2005) Resistin is an inflammatory marker of atherosclerosis in humans. Circulation 111:932-939

Schwartz DR, Lazar MA (2011) Human resistin: found in translation from mouse to man. Trends Endocrinol Metab 22:259-265. doi:10.1016/j.tem.2011.03.005

Silswal N, Singh AK, Aruna B, Mukhopadhyay S, Ghosh S, Ehtesham NZ (2005) Human resistin stimulates the proinflammatory cytokines TNF-alpha and IL-12 in macrophages by NF-kappaB-dependent pathway. Biochem Biophys Res Commun 334:1092-1101

Solfrizzi V, D'Introno A, Colacicco AM, Capurso C, Todarello O, Pellicani V, Capurso SA (2006) Circulating biomarkers of cognitive decline and dementia. Clin Chim Acta 364:91-112. doi:10.1016/j.cca.2005.06.015

Song J, Lee JE (2013) Adiponectin as a new paradigm for approaching Alzheimer's disease. Anat Cell Biol 46:229-234. doi:10.5115/acb.2013.46.4.229 
Swarbrick MM, Havel PJ (2008) Physiological, pharmacological, and nutritional regulation of circulating adiponectin concentrations in humans. Metab Syndr Relat Disord 6:87-102. doi:10.1089/met.2007.0029

Tan ZS, Beiser AS, Vasan RS, Roubenoff R, Dinarello CA, Harris TB, Benjamin EJ, Au R, Kiel DP, Wolf PA, Seshadri $S$ (2007) Inflammatory markers and the risk of Alzheimer disease: the Framingham Study. Neurology 68:1902-1908. doi:10.1212/01.wnl.0000263217.36439.da

Teixeira AL, Diniz BS, Campos AC, Miranda AS, Rocha NP, Talib LL, Gattaz WF, Forlenza OV (2013) Decreased levels of circulating adiponectin in mild cognitive impairment and Alzheimer's disease. Neuromolecular Med 15:115-121. doi:10.1007/s12017-012-8201-2

Teunissen CE, Van der Flier WM, Scheltens P, Duits A, Wijnstok N, Nijpels G, Dekker JM, Blankenstein RM, Heijboer AC (2015) Serum leptin is not altered nor related to cognitive decline in Alzheimer's disease. J Alzheimers Dis 44:809-813. doi:10.3233/JAD-141503

Tezapsidis N, Johnston JM, Smith MA, Ashford JW, Casadesus G, Robakis NK, Wolozin B, Perry G, Zhu X, Greco SJ, Sarkar S (2009) Leptin: a novel therapeutic strategy for Alzheimer's disease. L Alzheimers Dis 16:731-740

Theodoropoulou A, Metallinos IC, Psyrogiannis A, Vagenakis GA, Kyriazopoulou V (2012) Ghrelin and leptin secretion in patients with moderate Alzheimer's disease. J Nutr Health Aging 16:472-477
Tilg M, Moschen AR (2006) Adipocytokines: mediators linking adipose tissue, inflammation and immunity. Nat Rev Immunol 6:772-783. doi:10.1038/nri1937

Une K, Takei YA, Tomita N, Asamura T, Ohrui T, Furukawa K, Arai $\mathrm{H}$ (2011) Adiponectin in plasma and cerebrospinal fluid in MCI and Alzheimer's disease. Eur J Neurol 18:1006-1009. doi:10.1111/j.1468-1331.2010.03194.x

van Himbergen TM, Beiser AS, Seshadri S, Otokozawa S, Au R, Thongtang N, Wolf PA, Schaefer EJ (2012) Biomarkers for insulin resistance and inflammation and the risk for allcause dementia and Alzheimer disease: results from the Framingham Heart Study. Arch Neurol 69:594-600. doi:10.1001/archneurol.2011.670

Warren MW, Hynan LS, Weiner MF (2012) Lipids and adipokines as risk factors for Alzheimer's disease. J Alzheimers Dis 29:151-157. doi:10.3233/JAD-2012111385

Wellen KE, Hotamisligil GS (2003) Obesity-induced inflammatory changes in adipose tissue. $\mathrm{J}$ Clin Invest 112:1785-1788

Zoico E, Di Francesco V, Mazzali G, Vettor R, Fantin F, Bissoli L, Guariento S, Bosello O, Zamboni M (2004) Adipocytokines, fat distribution, and insulin resistance in elderly men and women. J Gerontol A Biol Sci Med Sci 59:M935M939 